\title{
Paraguay revisité par Rennes : à propos de quelques publications récentes
}

\section{Carmen Bernand}

\section{(2) OpenEdition}

\section{$\checkmark$ Journals}

Édition électronique

URL : https://journals.openedition.org/jsa/11995

DOI : 10.4000/jsa. 11995

ISSN : 1957-7842

\section{Éditeur}

Société des américanistes

\section{Édition imprimée}

Date de publication : 20 décembre 2011

Pagination : 403-410

ISSN : 0037-9174

\section{Référence électronique}

Carmen Bernand, «Paraguay revisité par Rennes : à propos de quelques publications récentes ", Journal de la Société des américanistes [En ligne], 97-2 | 2011, mis en ligne le 22 décembre 2011, consulté le 04 septembre 2022. URL : http://journals.openedition.org/jsa/11995 ; DOI : https:// doi.org/10.4000/jsa.11995

Ce document a été généré automatiquement le 4 septembre 2022.

Tous droits réservés 


\title{
Paraguay revisité par Rennes : à propos de quelques publications récentes
}

\author{
Carmen Bernand
}

\section{RÉFÉRENCE}

À propos des ouvrages suivants : BoIDIN Capucine, 2011, Guerre et métissage au Paraguay, 2001-1767, Presses Universitaires de Rennes, Rennes ; CAPDEVILA Luc, 2007, Une guerre totale : Paraguay, 1864-1870. Essai d'histoire du temps présent, Presses Universitaires de Rennes, Rennes; CAPDEVILA Luc, Isabelle COMBÈs, Nicolas RICHARD et Pablo BARBOSA, 2010, Les hommes transparents. Indiens et militaires dans la guerre du Chaco (1932-1935), Presses Universitaires de Rennes, coll. « Histoire », Rennes ; RICHARD Nicolas (éd.), 2008a, Mala guerra. Los indígenas en la guerra del Chaco (1932-1935), ServiLibro/Museo del Barro/CoLibris, Asunción del Paraguay ; RICHARD Nicolas, 2008b, Les chiens, les hommes et les étrangers furieux. Archéologie des identités indiennes dans le Chaco boréal, thèse de doctorat, EHESS, Paris [non publiée] ; WILDE Guillermo, 2009, Religión y poder en las misiones de guaranies, SB, Buenos Aires.

1 Dans un pays aussi centralisé que la France, l'idée que des problématiques américanistes nouvelles puissent surgir ailleurs que dans le cocon des prestigieux laboratoires parisiens, semble improbable. Et, pourtant, une anthropologie différente, centrée sur l'événement, a trouvé autour de Luc Capdevila, professeur d'histoire à l'université de Rennes, et de l'équipe de recherche qu'il a constituée, l'émulation intellectuelle nécessaire pour aborder des thématiques originales. Le terrain qui fédère ces chercheurs, venus d'horizons géographiques et disciplinaires divers, c'est le Paraguay. Leurs travaux ont bénéficié d'une large diffusion de la part des Presses Universitaires de Rennes, qui jouent un rôle de premier plan dans ce renouveau de l'américanisme. 
2 Laissant aux historiens les métissages et les maisonnées polygamiques du «paradis de Mahomet ", comme les Espagnols du Xvi siècle appelaient Asunción et ses environs, boudant les réductions guarani, pourtant une expérience politique et culturelle majeure, en raison de leur forte acculturation religieuse ${ }^{1}$, l'anthropologie s'est focalisée sur le Chaco boréal, considéré comme un réceptacle de peuples archaïques, à l'abri des contingences historiques en raison de son isolement. Moi-même, il y a déjà de nombreuses décennies dans ma thèse sur les Ayoré, j'ai succombé à l'attrait de l'altérité totale que représentaient, pour moi, la région et ses peuples.

3 S'il y a donc un lieu qui a incarné, jusqu'à une époque récente, le monde premier, primordial ou primitif de l'Amérique du Sud, c'est bien le Chaco, dont le territoire géographique et ethnique déborde d'ailleurs des frontières nationales pour s'étendre en Bolivie orientale, en Argentine septentrionale et sur une frange du Brésil. Encore faut-il s'entendre sur le bornage par l'État-nation d'une contrée dont la démarcation était bien difficile à tracer. Dès l'époque coloniale, la question des démarcations est primordiale pour la survie des nations originaires du Paraguay - le terme de nation est ici employé dans son acception ancienne. En premier lieu, il y a la ligne, imprécise sur le terrain mais implacable dans la juridiction, qui sépare depuis 1494 les possessions portugaises de celles de l'Espagne, et qui concerne directement les indigènes, libres de ce côté-ci, esclaves de ce côté-là. Puis, la barrière géographique, juridique et politique qui se dresse entre le Paraguay et le Piémont de la Cordillère des Andes (aujourd'hui la Bolivie). Entre les deux ensembles, s'étend une sorte de no man's land traversé par des harcèlements récurrents des Indiens, des militaires et des colons. Enfin, une fracture sépare, dès le début du XVII e siècle, le Paraguay et le Rio de la Plata, unis jusque-là par une histoire commune et libérant pour longtemps un autre no man's land sur les rives du Pilcomayo. Ces frontières coloniales ont laissé des traces dans la reconstitution des groupes ethniques et dans la mémoire.

4 Dans le Chaco boréal, les territoires indigènes, soumis à leur propre contingence, subissent aussi les conséquences de la Conquête. Malheureusement inédite, la thèse de Nicolas Richard (2008b) montre de façon éclairante les transformations des ethnonymes en fonction des rapports entre groupes dominants et tribus périphériques. Les conquérants et les missionnaires glanent ces noms de leurs propres informateurs et alliés. Cette nomenclature sera par la suite adoptée par l'anthropologie. D'où la difficulté, quand on veut reconstituer la mosaïque des populations, de se frayer un chemin à travers cette cacophonie d'appellations. Le travail de Richard ne se borne pas à ce constat. En suivant dans les documents historiques la trace des contacts et à force de recoupements, il réussit à reconstituer l'histoire des contacts, des réseaux et des migrations de tous ces peuples, réputés pourtant en marge de l'histoire.

5 Après l'indépendance du Paraguay, les frontières nationales ont été menacées à deux reprises: entre 1864 et 1870, par la guerre de la Triple Alliance et, au xx siècle, par la guerre du Chaco, qui a duré de 1932 à 1935. Sur ce qui fut une guerre totale, opposant le Paraguay à la coalition formée par le Brésil, l'Argentine et l'Uruguay, Luc Capdevila a publié un ouvrage de référence qui rompt avec l'histoire officielle ${ }^{2}$. Au terme de ce conflit, le Paraguay perdit plus de $60 \%$ de sa population et devint - c'est le fondement même du récit - un pays sans hommes. La guerre du Chaco a donné lieu à deux ouvrages collectifs ${ }^{3}$. Enfin, la publication toute récente de Capucine Boidin (2011), sur les paysans paraguayens, pose le problème de la mémoire. Je me bornerai ici à présenter quelques points forts des livres publiés par les PUR afin de faire ressortir leur intérêt pour 
l'anthropologie américaniste. L'exercice est délicat car chacun de ces ouvrages est une mine d'informations. En dégager quelques aspects implique de laisser de côté des développements importants, que les lecteurs pourront d'ailleurs découvrir avec profit.

\section{Les Indiens et la guerre}

6 Le fil chronologique, qui correspond d'ailleurs aux dates de parution des ouvrages, débute avec Luc Capdevila. Les préliminaires de la guerre de la Triple Alliance doivent être cherchés dans l'expansion du Brésil, encore un empire, et de l'Argentine, avec un arrière-plan de rivalités pour le contrôle de la navigation fluviale, les marchandises et la militarisation des pays concernés. En ce qui concerne le Paraguay et ses populations autochtones, l'année 1848 est importante puisqu'elle correspond à la suppression légale, par le président Carlos Antonio López, des communautés indiennes guarani, déjà bien ébranlées par l'expulsion des Jésuites au siècle précédent. Les " peuples historiques " deviennent des citoyens. Quand la guerre de la Triple Alliance éclate, les Guarani sont donc appelés à servir sous le drapeau national. De même pour d'autres groupes, comme les Guaycuru Payagua qui avaient un statut particulier et étaient déjà en voie de prolétarisation. Cinq cents Payagua, qui avaient, jusqu'à une époque très proche, sillonné le fleuve Paraguay pour intercepter les bateaux étrangers furent recrutés par le gouvernement. Mais, de l'autre côté de la frontière, en Argentine, d'autres Guaycuru comme les Mocovi furent mobilisés par l'armée. Ceux-ci voulaient en découdre avec leurs voisins et trouvèrent l'occasion de venger des anciennes offenses tribales. Enfin, le Brésil fit de même avec les Guaycuru Mbayá ou Caduveo. C'est dire l'importance des bouleversements subis par ces populations. La géopolitique indigène fut complètement déstabilisée par le conflit et les Guaycuru, décimés, cessèrent de rançonner les tribus périphériques. Les fiers Caduveo que Claude Lévi-Strauss immortalisa dans Tristes Tropiques, avaient encore leurs peintures faciales, mais celles-ci n'avaient plus qu'une signification esthétique. Le contexte qui leur donnait sens avait disparu depuis longtemps.

7 Bien entendu, les Indiens ne sont pas les seuls protagonistes de cette histoire, qu'ils partagent avec d'autres groupes. Sans entrer ici dans les détails de cet ouvrage, signalons quelques thèmes développés par la campagne belliciste. Car la Guerre passe aussi par les images de propagande publiées dans le journal Cabichui et reproduites partiellement par l'auteur. Le Brésil apparaît comme un pays de Noirs aux traits simiesques accentués. L'impératrice elle-même a des traits africains. La femme paraguayenne accompagne les soldats, ranime les vieillards et stimule leur sexualité, au service de futurs concitoyens. Ces xylogravures se trouvent conservées à Asunción, dans le Museo del Barro et attendent leur ethnologue. Elles ont été exposées à Paris, à l'ambassade du Paraguay, en 2005.

8 La guerre du Chaco n'est pas seulement un conflit patriotique entre deux États souverains, la Bolivie et le Paraguay. Elle s'inscrit dans un cycle de confrontations ouvertes par le " colonialisme républicain » contre les derniers réduits de "barbarie », espaces sauvages qu'il faut intégrer à la civilisation. Dans cette wilderness australe qu'il faut incorporer au territoire national et dont l'équivalent espagnol est desierto (despoblado à l'époque coloniale), vivent plus de 50000 Indiens, pour la plupart des chasseurscueilleurs, mais aussi des agriculteurs comme les Chané et les Chiriguano. Le Paraguay veut donc stabiliser la présence de l'État dans la région. Il considère que les propriétaires 
légitimes de ce " désert » sont les Guarani et les Guaycuru, citoyens paraguayens. Pour la Bolivie, les Indiens du Chaco appartiennent à la " race indigène ", comme les Aymara et les Quechua et, par conséquent, ils ont leur place dans ce pays. L'identité nationale des Indiens du Chaco sera façonnée par la guerre. Il y a là matière à réflexion pour tous ceux qui s'intéressent à l'ethnicité.

9 L'étincelle qui met le feu aux poudres et inaugure la guerre du Chaco est l'occupation de la lagune Pitiantuta, «le lieu du fourmilier mort », par un détachement bolivien qui fonce sur le petit poste militaire paraguayen, perdu dans une contrée aride. Pitiantuta était avant tout un lieu à l'existence conservée par la tradition orale et dont l'accès n'était connu que par quelques éclaireurs indigènes. Des tribus zamuco habitaient à proximité. Ce centre vital pour la guerre, puisque c'est le seul réservoir d'eau de toute la région, est atteint par deux colonnes. L'une est menée par le colonel bolivien Ayoroa, l'autre est conduite par un Russe tsariste, Juan Belaief, pour le compte du Paraguay. Dans les deux cas, les éclaireurs indigènes s'avérèrent indispensables; dans les deux cas, les relations entre les militaires et les autochtones furent amicales. Il est possible que le colonel Ayoroa soit à l'origine du nom Ayoré pris par les bandes Zamuco-Moro dans les années 1940.

Aux côtés de Belaief, personnage d'une truculence romanesque, se trouvent deux caciques chamacoco, Capitán Pinturas et Chicharrón, qui sont des guides remarquables. À la faveur des déplacements militaires paraguayens, ces caciques comptent bien récupérer des territoires perdus, dont la célèbre lagune - ce qui montre d'ailleurs les liens entre Zamuco-Ayoré et Chamacoco de la rive du Paraguay. D'autres caciques, qu'ils soient Nivaklé ou Chiriguano, interviennent dans la guerre, poursuivant leurs propres objectifs. Les auteurs des Hommes transparents - les Indiens, oubliés par l'histoire officielle parviennent à reconstituer les généalogies et à introduire l'histoire dans le tableau intemporel des tribus du Chaco. Ils rouvrent le débat lancé par Pierre Clastres sur les néoprimitifs, en y apportant une documentation historique et ethnographique. À la lecture de ces aventures, les élucubrations sur l'Autre, chères à l'anthropologie de ces dernières décennies, paraissent bien creuses. Non seulement ici, et dans des circonstances très pénibles, la communication entre les différents acteurs ne pose pas de problème, mais encore on peut se demander lequel de Belaief ou de Chicharrón incarne cette altérité réifiée...

11 Partie au Paraguay pour étudier les paysans guarani de San Ignacio et ses hameaux, Capucine Boidin sent très vite que son projet initial s'enlise, puis bifurque dans une direction insoupçonnée. Car le vrai sujet qui s'offre à elle, celui qui occupe les conversations et sur lequel convergent récits et pratiques, histoires et recettes de cuisine, est bien la Guerre, celle de La Triple Alliance. Paradoxalement, c'est précisément le sujet que l'on ne peut pas décrire puisqu'il a été évacué de la mémoire, et toutes les données ethnographiques viennent s'ordonner autour de ce point noir.

La singularité de ce livre réside dans l'effort conjoint et souvent contradictoire de son auteur et de ses interlocuteurs pour recoudre la déchirure du temps. Cette expérience correspond à ce que Hannah Arendt et François Hartog appellent une "brèche ». Cette faille survient à la suite d'événements dont l'intensité destructrice empêche le peuple qui la subit de renouer avec sa vie d'avant. Pour repartir, il faut faire table rase du passé. Cette séparation ébranle les fondements même de la personne humaine, son identité, ses relations familiales, ses racines, ses appartenances, et l'anthropologie, attachée davantage aux permanences qu'aux discontinuités, ne l'avait pas vraiment envisagée. 
Guerre et métissage au Paraguay... ne dénonce pas, mais noue patiemment les fils coupés. Les questions posées par Capucine Boidin à ses hôtes buttent sur une date, 1870. Les informatrices reprennent le récit $\mathrm{du}$ " pays sans hommes ». La vie a pu reprendre, disentelles, grâce à l'arrivée de migrants argentins - originaires donc d'un État agresseur. Les métissages qui en résultent nécessairement tissent progressivement la nation à partir des lambeaux de la défaite.

La guerre est donc une brèche dans l'histoire et dans la mémoire des gens. À Isla Guasu, explique une octogénaire, au lendemain du conflit, il ne restait plus qu'un tas d'herbes et un jaguar qui rôdait et dévorait les malheureuses femmes qui s'aventuraient hors de chez elles. Alors que ce félin est le prédateur par excellence - la mythologie amérindienne le répète de façon récurrente - dans ce récit de l'année zéro, le jaguar avait été auparavant un Indien qui avait refusé d'aller à la guerre (un lâche ?) et qui se nourrissait désormais de la chair des femmes, avant d'être tué à son tour, sacrifice indispensable pour qu'une nouvelle vie puisse reprendre. Dans cette situation sidérante qui est celle de la cessation des combats par mort généralisée, le paradigme de l'origine des hommes, que tous les mythes reconnaissent dans la différentiation progressive des humains et de leurs pratiques culinaires, est renversé puisque l'homme qui s'est soustrait à la prédation de la guerre, devient animal.

indéterminée, de cette brèche impossible à combler, datent d'autres rêves typiques de situations extrêmes, comme la quête vaine de trésors enfouis ou les indiscrétions de perroquets trop bavards. Ces histoires, comme le souligne Capucine Boidin, ne sont pas vraiment des mythes, dans le sens strict du terme, mais des récits mythifiés, comme si ce genre hybride était la seule façon possible de parler de l'indicible. Cette mémoire imagée ne constitue donc pas un mythe d'origine, puisque rien n'est créé, c'est plutôt le récit de l'effacement même des racines humaines.

Les généalogies, les rapports de parenté et d'alliance, les installations successives dans différents terrains sont toujours une aide pour donner une épaisseur temporelle aux relations humaines. Ici, ces constructions, qui agrémentent le dialogue entre l'anthropologue et les paysans, ne permettent pas de tendre une passerelle entre les deux rives du passé. En vain, Capucine Boidin recourt aux archives, assez peu loquaces, d'ailleurs, sur ces questions, pour stimuler les souvenirs des uns et des autres. La mémoire semble solidement verrouillée et la faille qui sépare le jadis et le présent est infranchissable: aux informations précises que l'anthropologue glane dans les documents, ses hôtes lui opposent un déni poli. Tous les hommes du Paraguay sont morts, répète-t-on. Ne sont restées en vie que les femmes, et elles ont dû épouser des étrangers. Avec eux sont arrivés aussi les vaches, exterminées comme les humains, et le repeuplement a commencé.

16 Ces convictions solidement chevillées au corps sont-elles des «représentations collectives » ou ont-elles été inculquées par la propagande officielle? Avec subtilité Capucine Boidin dénoue l'écheveau de la manipulation politique, pratiquée à l'époque par le parti libéral. Car même si la mortalité a été très élevée, il y a eu, bien évidemment, des survivants. Mais il était pressant pour la nation d'effacer la honte de la défaite et, pour cela, il fallait balayer de la mémoire collective l'existence des vétérans, qui furent ainsi condamnés à la mort sociale. Aucun héros vivant n'émerge du désastre, et les vétérans deviennent dans les remémorations des étrangers, voire des Espagnols... Dépossédés de leur honneur et de leur identité, ils deviennent littéralement « autres » et, 
à ce prix, acceptables et acceptés. Ils ne seront pleinement reconnus par le gouvernement qu'à la fin du XIX ${ }^{e}$ siècle.

17 Le terme culinaire de jopara est, pour les habitants de la région de San Ignacio, une métaphore de leur langue. Leur idiome jopara est en fait un bilinguisme, sans que cela implique une fusion de ses deux composantes, la langue indigène guarani et l'espagnol. Les étrangers (provenant de Corrientes, en Argentine) et les Indiens parlent le guarani, les autres, le castillan. Entre les deux, les «jopara parlants» occupent une place à part, dominée certes, mais susceptible de s'introduire dans les deux mondes. Ce troisième terme n'est pas vraiment une synthèse de deux opposés, mais un atout, celui des gens " interstitiels ", à l'écart des normes imposées par ceux qui ont le pouvoir et qui peuvent déployer des tactiques diverses au gré des situations.

Il reste pourtant une dernière brèche à explorer et Capucine Boidin réussit un tour de force en replaçant la borne temporelle en 1767, date de l'expulsion des Jésuites. L'enquête suit l'histoire tortueuse des hameaux de San Ignacio, désignés par les paysans comme des " compagnies» rurales, mais invisibles sous ce nom dans les archives. Au terme d'une enquête compliquée en raison de l'opacité des références ethniques, Capucine Boidin reconstruit avec brio l'itinéraire des Indiens « libérés » des réductions, que l'on croyait retournés dans la forêt. Comme la « lettre volée » d'Edgard Poe, évidente et, à cause de cela, invisible au regard, les descendants des Guarani de la réduction sont bien sous ses yeux, dans les espaces interstitiels des compagnies rurales, sauf qu'ils ne se disent plus indigènes, mais métis jopara. La plupart d'entre eux n'ont plus de terre et ont été recueillis par des parents plus fortunés. À Isla Guasu les plus pauvres s'entassent dans le quartier de la Chacarita sans pouvoir quitter leurs maisons quand survient un décès, comme la coutume le veut. En s'incrustant sur les lieux malgré eux, ils «ferment les chemins ", qui ne s'ouvrent que sur les ruines des maisons abandonnées. La Chacarita est d'ailleurs le nom du plus grand cimetière de Buenos Aires, une ville dans la ville, que tous les migrants paraguayens connaissent. Coïncidence ou plutôt analogie métaphorique? Plus les maisons s'éloignent les unes des autres, plus leurs habitants ont tendance à délaisser le parler jopara, à acquérir des titres de propriété et à occuper une position de pouvoir par rapport aux «tolérés ». Ces hiérarchies ne sont pas fixes, mais fluctuantes, travaillées par la politique et par le clientélisme, qui pallie les carences de l'État. Le métissage, avec les systèmes symboliques qui le sous-tendent, n'est compréhensible qu'au sein de relations de pouvoir inscrites dans une longue histoire, déroulée ici de façon régressive à partir de la vie quotidienne des familles. Mais, contrairement à l'idée générale sur la brèche ouverte par la guerre, ce livre montre in fine le poids du passé dans la construction des espaces interstitiels d'où surgissent des luttes pour ouvrir de nouveaux chemins.

19 La richesse des ouvrages que nous venons d'évoquer réside dans la pluridisciplinarité des approches. Sauf Luc Capdevila, qui est incontestablement historien, les autres auteurs ont été formés à l'anthropologie et à la sociologie, mais la logique du terrain qu'ils ont choisi les a menés à faire aussi de l'histoire. En somme, ils ont construit une anthropologie de l'événement, en analysant avec beaucoup de finesse les registres d'historicité en présence. Ils ont également évité les schémas raciaux qui sous-tendent les prémisses de l'altérité. Les Indiens sont avant tout des acteurs, des gens qui font l'histoire, au même titre que le colonel Ayoroa ou Juan Belaief, et, au-delà, les présidents et les états-majors. Enfin, en analysant les stratégies à l'œuvre, les alliances, les trahisons, les compromissions ou les défections, ces auteurs laissent à découvert, sans 
l'avoir voulu probablement, les limites de cette vieille notion de résistance indienne que nous avons assumée avec enthousiasme, projetant sur les peuples «transparents » nos aspirations et nos fantasmes.

\section{NOTES}

1. Wilde (2009). Ce travail remarquable d'anthropologie historique a reçu en 2010 le prix du LASA, pour le meilleur livre dans ce domaine.

2. Capdevila (2007). Une traduction en espagnol a vu le jour en 2010, à Buenos Aires.

3. Voir Richard (2008a) et Capdevila et al. (2010).

\section{AUTEURS}

\section{CARMEN BERNAND}

Professeur émérite, Université Paris Ouest Nanterre La Défense 